\title{
Determining the Adsorption and Desorption Properties of Flavonoids Found in Eucommia ulmoides Oliv. Leaves Using Macroporous Resin and Acetylcholinesterase Inhibitor Screening
}

\author{
Zhihong Wang, ${ }^{\mathrm{a}, \mathrm{b}}$ Zhigang She, ${ }^{\mathrm{a}, *}$ Mijun Peng, ${ }^{\mathrm{b}, *}$ Qiuling Yang, ${ }^{\mathrm{b}}$ and Tao Huang ${ }^{\mathrm{b}}$
}

The adsorption and desorption properties of 12 resins containing flavonoid compounds found in Eucommia ulmoides Oliv. leaves (EUOL) extracts were investigated. The static adsorption and desorption, kinetic, adsorption, and thermodynamic properties of the adsorption of the flavonoids onto macroporous resins were determined. The HPD-300, NKA-9, and AB-8 resins exhibited a greater adsorption capacity and desorption characteristics. A pseudo-second-order kinetic model was suitable to characterize the kinetics of the adsorption of flavonoids onto the resins selected, and the diffusion of flavonoids was divided into three stages, with the boundary layer diffusion and intra-particle diffusion being the rate-controlling factors. The Langmuir model was found to be the best description of the adsorption behavior of flavonoids. Thermodynamic studies indicated that the adsorption of flavonoids was a physical, exothermic, and spontaneous process. The $60 \%$ ethanol eluted fraction from the NKA-9 resin column not only had the highest flavonoid content, but also possessed the strongest inhibitory effect on acetylcholinesterase. In addition, the degree of binding of the main flavonoid compounds found in the EUOL to acetylcholinesterase compounds was investigated via molecular docking technology. The results showed that the docking total score of isoquercetin and enzyme proteins were the highest, followed by kaempferol-3-O-rutinoside.

Keywords: Eucommia ulmoides Oliv. leaves; Flavonoids; Enrichment; Adsorption characteristics; Acetylcholinesterase inhibitors

Contact information: a: School of Chemistry, Sun Yat-Sen University, Guangzhou 510275 China; b: Guangdong Provincial Key Laboratory of Emergency Test for Dangerous Chemicals, Guangdong Institute of Analysis (China National Analytical Center Guangzhou), Guangdong Academy of Sciences, Guangzhou 510070 China; *Corresponding authors: cesshzhg@mail.sysu.edu.cn; pengmj163@163.com

\section{INTRODUCTION}

Resources from Eucommia ulmoides Oliv. are abundant in China, and mainly distributed in Hunan, Henan, Shaanxi, and Guizhou providence. Eucommia ulmoides has high application value in edible, medicinal, and ornamental characteristics. Its leaves, stem, bark, and even the staminate flower have high development prospects (He et al. 2014; Hirata et al. 2014; Zhu and Sun 2018). It is noteworthy that the bark of woody plants is usually rich in active ingredients and has potential biological activity (Tanase et al. 2018, 2019), E. ulmoides bark is widely used as a valuable medicinal material, and it can be used alone or mixed with other substances for anti-aging, anti-Alzheimer's disease, or anti-tumor treatments; it also exhibits antioxidant properties and prevents hypertension, hyperlipidemia, hyperglycemia, and osteoporosis, as well as providing immune system 
enhancement (Kwon et al. 2011; He et al. 2014; Luo et al. 2014; Bai et al. 2015; Li et al. 2017; Hosoo et al. 2017; Cho et al. 2018; Zhu and Sun 2018). However, the collection of E. ulmoides bark is often affected by the growth cycle and resources, so its applications are limited (Zhu et al. 2016). The results of research in recent years have shown that $E$. ulmoides leaves (EUOL) have a similar chemical composition to the bark, and that EUOL are richer in resources, have shorter growth cycles, and are easier to collect (Zhu et al. 2016, 2018). Moreover, EUOL were not only listed in the Chinese Pharmacopoeia, they are used as an important substance for homology of medicine and food in China in 2020 (State Pharmacopoeia Commission of the PRC 2015). EUOL have broad application prospects in the food and pharmaceutical industries. Therefore, the development and utilization of EUOL with abundant yield and low price will have certain economic value and social benefits.

Modern pharmacological investigations indicate that EUOL primarily contain flavonoids, polyphenols, and iridoids (Kim et al. 2004; He et al. 2014; Hirata et al. 2014). In addition to having similar effects to E. ulmoides bark, they also have anti-inflammatory, anti-diabetic, sedative, and weight loss effects (Lee et al. 2005; Zhou et al. 2009; Zhang et al. 2012; Li et al. 2013). Presently, the research of natural products is primarily focused on the discovery, preparation, efficacy evaluation, and product development of active ingredients and components. Therefore, the rapid preparation of the active ingredients in EUOL, the evaluation of biological activities, the confirmation of target substances, and the mechanism of action have always been the focus of attention. As a potential feedstock, EUOL have rich natural bioactive ingredients with multiple functions for healthcare, which has prompted the development and utilization of E. ulmoides as a human health food, drug, and animal feed additive (Xu et al. 2010; Zhu and Sun 2018). Among them, flavonoids are the main compound class found in EUOL (Xing et al. 2019). With the increase in the number of reports on the application and development of flavonoids to human health, flavonoids of EUOL have also attracted interest. Flavonoids contain phenyl and hydroxyl groups, which affect the polarity of the compound. In addition, the polarity difference between flavonoid aglycone and flavonoids containing glycosides will be more obvious (Tanase et al. 2019). Therefore, it is necessary to select different polar resins to enrich flavonoids. And due to their complex composition, their content is usually measured using the colorimetric method (Hou et al. 2020). The composition of flavonoids has been explored, and some flavonoids have been discovered and identified, but it is difficult to achieve complete clarity. The flavonoid compounds that have been discovered are difficult to achieve complete separation via traditional high performance liquid chromatography (HPLC) due to their multiple flavonoid aglycones and glycoside types and their complex structures (He et al. 2014). It is worth nothing that the results of in vivo and in vitro experiments showed that the EUOL extracts can prevent Alzheimer's disease (AD) (Zhou et al. 2009). In clinics, acetylcholinesterase (AChE) inhibitors have been used to screen for the treatment of AD. The screening model for this inhibitor was relatively simple and easy to operate (Wu et al. 2020). Since flavonoids are abundant in EUOL, it was also meaningful to use this model to screen for active components with an enzyme inhibitory effect (Hirata et al. 2014). Therefore, in order to improve the utilization value of the flavonoids extracted from EUOL, the preparation and activity evaluation of flavonoid fractions from EUOL needed to be investigated.

In the field of green chemistry, the utilization of biomass resources has attracted special interest. Obtaining biologically active compounds that can be used in different fields through efficient and green methods is an important topic that needs to be explored 
all the time (Tanase et al. 2018). In this process of obtaining the target substance, undoubtedly, the enrichment of biologically active compounds from crude plant extracts is a very critical step, which is of particular significance for the comprehensive utilization of EUOL resources. Several techniques have been used to target compounds, e.g., chromatography using a silica gel column or Sephadex LH-20 column or ultrafiltration using specific membranes and liquid-liquid extraction with ethyl acetate. The main drawbacks of these techniques are that they can be complex, time-consuming, costly, harmful to the environment, or not suitable for industrial production systems (Jia and Lu 2008; Zhong et al. 2019). In response to the above mentioned, macroporous adsorption resin is a synthetic polymer material with good mechanical properties, chemical stability, and high adsorption capacity. This method is an excellent technology that can enrich biologically active compounds from crude plant extracts and has many unparalleled advantages, such as high efficiency, better selectivity, good mechanical properties, environmental protection, energy saving, low solvent consumption, and easy operation. More importantly, this approach can be more suitable for industrial applications (Jiang et al. 2020). Resin adsorption and desorption technology provides an ideal tool for enriching flavonoids. However, due to different interactions at the solid-liquid interface, it is difficult to accurately predict the adsorption capacity of the adsorbate/adsorbent system. Furthermore, the physical properties of the resin and the chemical properties of the solvent also play a role in the adsorption process. In the enrichment process, polarity is a key factor to be considered, and macroporous resins include polar, weakly polar and non-polar resins, which can be used for the enrichment of natural products. The hydrophobicity, hydrophilicity and functional groups of the resin also have an important influence on the properties of adsorbed substances (Jin et al. 2015). For this process, the macroporous resin binds to the target substance through non-covalent bonds (hydrogen bond and van der Waals force, etc.). In addition, different organic solvents can be used to desorb the adsorbate from the adsorbent. It is worth considering that the study of multi-component systems needs to understand every single component of the system. For these reasons, the adsorption and desorption mechanism of EUOL flavonoids on the macroporous resin still has many unknown problems to be explored. In order to understand the adsorption behavior of selected resins towards the target compounds, adsorption studies in aqueous solutions were performed. The primary aim of this work was to provide a technical and theoretical basis for the industrial application of EUOL.

The establishment of the macroporous resin enrichment technology mainly depends on the difference in the interaction between the resin and the target active substance. A suitable preparation method is essential for the efficient use of resources, and it was necessary to understand the adsorption and desorption mechanisms of the macroporous resins. Therefore, kinetic data could be fit to kinetic models (pseudo-first-order kinetics and pseudo-second-order kinetics models) to clarify the type of adsorption (Yang et al. 2016). Adsorption isotherm models, e.g., Langmuir or Freundlich, were chosen to speculate on the adsorption mechanisms, and additional thermodynamic parameters, i.e., enthalpy change, entropy change, and free energy, can be derived ( Lv et al. 2018).

Therefore, the objective of this work was to try to explore a simple, effective and practical method to enrich total flavonoids from EUOL through macroporous adsorption resin and determine the type of macroporous resin and operating conditions that yielded the greatest improvement on the purification of the target compounds extracted from EUOL. Adsorption kinetics, static adsorption and desorption, the adsorption isotherm, and thermodynamic adsorption parameters of the target compounds of interest would be 
evaluated. Different elution components were obtained via gradient elution from the selected resins, the flavonoid content of the sample was detected, and the acetylcholinesterase inhibitory effect of different samples was also evaluated. In order to further understand the binding effect of the EUOL flavonoids and enzymes, the degrees of docking were evaluated and compared via molecular docking techniques. Based on the above mentioned, this study systematically investigated the enrichment and activity evaluation of total flavonoids in EUOL, and the result will provide a valuable theoretical reference for the comprehensive utilization of E. ulmoides resource.

\section{EXPERIMENTAL}

\section{Materials and Chemicals}

The macroporous resins (HPD-100, HPD-300, HPD-600, D-3250, X-5, D-140, NKA-9, NKA-II, D-101, AB-8, S-8, and polyamide) were purchased from Zhengzhou Qinshi Technology Co., Ltd (Zhengzhou, China). The moisture content of each of the purchased microporous resins were determined. Before usage, the macroporous resins were pretreated by soaking in $95 \%$ ethanol ( 1 to 5 ratio, v/v) and eluted with $5 \% \mathrm{NaOH}$ (1 to 5 ratio, v/v) and $5 \% \mathrm{HCl}(1$ to 5 ratio, v/v) and finally thoroughly washed with deionized water. The physical properties of the macroporous resins were summarized in Table 1.

Table 1. Physical Characteristics of the Macroporous Resins

\begin{tabular}{|c|c|c|c|c|c|}
\hline Resins & Polarity & $\begin{array}{c}\text { Average Pore } \\
\text { Diameter } \\
\text { (nm) }\end{array}$ & $\begin{array}{c}\text { Partical Size } \\
\text { (mm) }\end{array}$ & $\begin{array}{c}\text { Specific Surface } \\
\text { Area }\left(\mathrm{m}^{2} / \mathrm{g}\right)\end{array}$ & $\begin{array}{c}\text { Pore Volume } \\
\text { (mL/g) }\end{array}$ \\
\hline HPD-100 & Nonpolar & 8.5 to 9.0 & 0.3 to 1.25 & 650 to 700 & 1.35 to 1.65 \\
\hline HPD-300 & Nonpolar & 5.0 to 5.5 & 0.3 to 1.2 & 800 to 870 & - \\
\hline HPD-600 & Polar & 8.0 & 0.3 to 1.2 & 550 to 600 & - \\
\hline D-3520 & Nonpolar & 8.5 to 9.0 & 0.3 to 1.25 & 480 to 520 & 2.10 to 2.15 \\
\hline X-5 & Nonpolar & 29 to 30 & 0.3 to 1.25 & 500 to 600 & 1.20 to 1.24 \\
\hline D-140 & Nonpolar & 9.5 & - & 500 to 600 & 1.00 to 1.50 \\
\hline NKA-9 & Polar & 15.5 to 16.5 & 0.315 to 1.25 & 170 to 250 & 1.00 to 1.04 \\
\hline NKA- I & Polar & 14 to 16 & 0.3 to 1.25 & 950 to 1250 & 0.62 to 0.66 \\
\hline D-101 & Nonpolar & 9 to 11 & 0.3 to 1.25 & 550 to 600 & 1.50 to 1.70 \\
\hline AB-8 & Weak polar & 13 to 14 & 0.3 to 1.25 & 480 to 520 & 0.73 to 0.77 \\
\hline S-8 & Polar & 28 to 30 & 0.3 to 1.25 & 100 to 120 & 0.78 to 0.82 \\
\hline Polyamide & Polar & - & - & 5 to 10 & - \\
\hline
\end{tabular}

Acetylcholinesterase, acetylthiocholine iodide (ACTI), and 5,5-dithiobis [2nitrobenzoic acid] (DTNB) were purchased from Sigma (St. Louis, MO). The rutin standard was purchased from Shanghai Yuanye Bio-Technology Co., Ltd (Shanghai, China). The other chemical reagents were analytical grade and were obtained from Sinopharm Chemical Reagent Company (Shanghai, China).

\section{Sample Preparation}

Fresh Eucommia ulmoides leaves were collected from Cili (Zhangjiajie, Hunan) during July 2019. All the plant raw materials were sterilized via a microwave oven and then dried at $55{ }^{\circ} \mathrm{C}$, and stored at $4{ }^{\circ} \mathrm{C}$ before use with voucher number 20190702. The dried sample was ground into a fine power using a laboratory mill (DFY-1000C, Wenling 
Linda Machinery Co., Ltd., Zhejiang, China) and stored at room temperature in a desiccator until used. The extraction of the EUOL compounds was carried out by following previous literature (Wang et al. 2020) with slight modifications. The milled samples were extracted with $50 \%$ ethanol (in a solvent ratio of 1 to $15(\mathrm{w} / \mathrm{v})$ ), and then evenly stirred, sonicated (at $50{ }^{\circ} \mathrm{C}$ for $30 \mathrm{~min}$ ) and filtered. Solvents were added to the residue after filtration, and the process was repeated three times. After that, all filtrates were collected, and the solvent was recovered via rotary evaporation (R-300, BUCHI Labortechnik AG, Flawil, Switzerland) under a partial vacuum at $60{ }^{\circ} \mathrm{C}$. The resulting yield was dry frozen (Alpha 2-4 LD plus, Marin Christ, Osterode, Germany) and stored in the fridge at $-20{ }^{\circ} \mathrm{C}$ for further use.

\section{Determination of the Total Flavonoid Content}

The total flavonoid content was determined using the method previously reported by Meda et al. (2005) with some modification. An appropriate amount of sample was added to the colorimetric tube, and $2.0 \mathrm{~mL}$ of $\mathrm{AlCl}_{3}$ was used to react with the flavonoids present in the samples, which was then diluted with $70 \%$ ethanol solution to bring the total volume up to $6.0 \mathrm{~mL}$. The absorbance was measured at $408 \mathrm{~nm}$ after allowing the reaction to run for $10 \mathrm{~min}$. The total flavonoid content was determined using a standard curve with rutin as the standard, and the calibration curve was obtained $(Y=0.0259 X-0.0133)$ and the linear correlation coefficient was $\mathrm{R}^{2}=0.9999$.

\section{Static Adsorption and Desorption Investigation}

The static adsorption capacities of the purchased resins (HPD-100, HPD-300, HPD600, D-3250, X-5, D-140, NKA-9, NKA-II, D-101, AB-8, S-8, and polyamide) were evaluated by the following procedure. The sample solutions were prepared by mixing the extract with the solvent in a proportion unique to each resin. An amount of fresh resin (equivalent to $1.0 \mathrm{~g}$ of dry resin) was weighed and added to a $150 \mathrm{~mL}$ flask, followed by $50 \mathrm{~mL}$ of the previously prepared sample solutions. The flasks were then shaken (at 120 $\mathrm{rpm}$ ) for $24 \mathrm{~h}$ at $25^{\circ} \mathrm{C}$ to reach the adsorption equilibrium. Then the resin was filtered with a Büchner funnel, and the total flavonoid content in the filtrate solution was determined via the colorimetric method. Next, the residual solution on the surface of the resin was washed off with deionized water, and different concentrations of an ethanol aqueous solution $(40 \%, 70 \%$, and $100 \%)$ were used for desorption. The total content of the target compounds in the desorbed solution were also analyzed via the above method. The equations used were presented as Eq. 1 (adsorption capacity), Eq. 2 (adsorption ration), and Eq. 3 (desorption ratio),

$$
\begin{aligned}
& Q_{t}=\frac{\left(C_{0}-C_{t}\right) V_{i}}{W} \\
& E(\%)=\frac{C_{0}-C_{t}}{C_{0}} \times 100 \% \\
& D(\%)=\frac{C_{d} V_{d}}{\left(C_{0}-C_{e}\right) V_{i}}
\end{aligned}
$$

where $Q_{\mathrm{e}}$ is the adsorption capacity at equilibrium ( $\mathrm{mg} / \mathrm{g}$ of dry resin), $D$ is the desorption ratio (\%), $C_{0}$ is the initial flavonoid content in the solutions $(\mathrm{mg} / \mathrm{mL}), C_{\mathrm{e}}$ is the flavonoid content in the solutions at equilibrium $(\mathrm{mg} / \mathrm{mL}), V_{\mathrm{i}}$ is the initial sample solution volume $(\mathrm{mL}), W$ is the dry resin weight $(\mathrm{g}), C_{\mathrm{d}}$ is the flavonoid content in the desorption solution $(\mathrm{mg} / \mathrm{mL})$, and $\underline{V}_{\mathrm{d}}$ is the desorption solution volume (mL) (Yang et al. 2016; Lv et al. 2018). 


\section{Adsorption and Desorption Kinetics of Selected Resins}

The adsorption kinetics of the selected resins were determined as follows. First, 20 $\mathrm{mL}$ of the previously obtained sample solution, based on the above methodology, was added to a $100 \mathrm{~mL}$ flask loaded with one of the selected fresh resins (equivalent to $5.0 \mathrm{~g}$ of dry resin). Then, the flask was shaken at $25{ }^{\circ} \mathrm{C}$ with a thermostatic oscillator at $120 \mathrm{rpm}$. Ultraviolet analysis for each of the $1.0 \mathrm{~mL}$ resin samples were conducted at $0 \mathrm{~min}, 5 \mathrm{~min}$, $15 \mathrm{~min}, 35 \mathrm{~min}, 55 \mathrm{~min}, 85 \mathrm{~min}, 115 \mathrm{~min}, 145 \mathrm{~min}$, and $175 \mathrm{~min}$. The kinetic adsorption curve was plotted using the total flavonoid content determined by the adsorption solution method described above. After reaching adsorption equilibrium, the resin was washed according to the previously described method. The desorption solvent was $60 \%$ ethanol, and the total flavonoid concentration of the sample was detected at the following time points: $0 \mathrm{~min}, 5 \mathrm{~min}, 15 \mathrm{~min}, 25 \mathrm{~min}, 45 \mathrm{~min}, 65 \mathrm{~min}, 95 \mathrm{~min}, 125 \mathrm{~min}$, and $155 \mathrm{~min}$. Based on the different flavonoid content in the solvents at different times, the desorption kinetic curve was also obtained. Simultaneously, to better understand the adsorption behaviors and mechanisms, pseudo-first-order and pseudo-second-order models were tested to predict the adsorption mechanism calculated using kinetic models. The kinetic parameters were calculated using Eq. 4 (pseudo-first-order kinetic model), Eq. 5 (pseudo-second-order kinetic model), and Eq. 6 (particle diffusion kinetics model),

$$
\begin{aligned}
& \ln \left(Q_{e}-Q_{t}\right)=-k_{1} t+\ln Q_{e} \\
& \frac{1}{Q_{t}}=\frac{1}{k_{2} Q_{e}^{2}} \cdot \frac{1}{t}+\frac{1}{Q_{e}} \\
& Q_{t}=k_{d} \cdot t^{1 / 2}+C
\end{aligned}
$$

where $Q_{\mathrm{e}}(\mathrm{mg} / \mathrm{g})$ is the adsorption capacity at equilibrium, $Q_{\mathrm{t}}(\mathrm{mg} / \mathrm{g})$ is the adsorption capacity at time $t, k_{1}\left(\mathrm{~min}^{-1}\right)$ is the rate constant of the pseudo-first-order, which is determined by plotting $\log \left(Q_{\mathrm{e}}-Q_{\mathrm{t}}\right)$ versus $t$ at various target compound concentrations, and $k_{2}$ ( $\left.\mathrm{g} / \mathrm{mg} \mathrm{min}\right)$ is the rate constant of the pseudo-second-order model, which is determined by plotting $1 / Q_{\mathrm{t}}$ versus $1 / t$, and $k_{\mathrm{d}}$ is the rate constant of particle diffusion (Wu et al. 2015).

\section{Adsorption Isotherms and Thermodynamics}

The different isotherm models were used to explain the adsorption relationship between the solid and liquid phases and further elaborate on the adsorption mechanism. A series of sample solutions were prepared; $10 \mathrm{~mL}$ of different concentration EUOL extract solutions were poured into the flasks and mixed with the pre-treated resins (equal to 0.50 $\mathrm{g}$ of dry resin). The flasks were incubated in a water bath shaker (at $120 \mathrm{rpm}$ ) at temperatures of $25^{\circ} \mathrm{C}, 35^{\circ} \mathrm{C}$, and $45^{\circ} \mathrm{C}$, respectively, until the absorption equilibrium was reached. Then the total flavonoid content of each solution was determined and the equilibrium concentration of the flavonoids for each flask was calculated.

The Langmuir equation and its variable form, described by Pompeu et al. (2010), are shown in Eq. 7 and Eq. 8,

$$
\begin{aligned}
Q_{e} & =\frac{q_{m} K_{L} C_{e}}{1+K_{L} C_{e}} \\
\frac{C_{e}}{Q_{e}} & =\frac{1}{Q_{m}} \cdot C_{e}+\frac{1}{q_{m} K_{L}}
\end{aligned}
$$

where $K_{\mathrm{L}}(\mathrm{g} / \mathrm{L})$ is the constant of the Langmuir model.

The Freundlich equation and its variable form, described by Wu et al. (2016), are shown in Eq. 9 and Eq. 10, 


$$
\begin{aligned}
& Q_{e}=K_{F} C_{e}^{1 / n} \\
& \ln Q_{e}=\frac{1}{n} \cdot \ln C_{e}+\ln K_{F}
\end{aligned}
$$

where $K_{\mathrm{F}}$ and $n$ are the constants of the Freundlich model.

The Sips model, described by Xiong et al. (2019), are shown in Eqs. 11 and 12,

$$
\begin{aligned}
& q_{e}=\frac{q_{m}\left(K_{S} C_{e}\right)^{\beta}}{1+\left(K_{S} C_{e}\right)^{\beta}} \\
& \frac{q_{m}}{q_{e}}=1+\frac{1}{\left(K_{S} C_{e}\right)^{\beta}}
\end{aligned}
$$

where $K_{\mathrm{FS}}(\mathrm{L} / \mathrm{g}), \alpha$, and $\beta$ are the Sips model constants.

Determining the adsorption thermodynamic properties is necessary to reveal the inherent energy change of the adsorbent after adsorption, and the mechanism involved in the adsorption process (Liu et al. 2016). The Gibbs free energy change $(\Delta G, \mathrm{~kJ} / \mathrm{mol})$, enthalpy change $(\Delta H, \mathrm{~kJ} / \mathrm{mol})$, and entropy change $(\Delta S, \mathrm{~J} /(\mathrm{mol} \cdot \mathrm{K}))$ are used to describe the SF adsorption thermodynamics at different temperatures. The enthalpy changes $(\Delta H)$ was calculated by the Van't Hoff equation, described by Wu et al. (2016), and shown in Eq. 13, Eqs. 14, and 15,

$$
\begin{aligned}
& \Delta G=-R T \ln K \\
& \Delta S=\frac{\Delta H-\Delta G}{T} \\
& \ln K=-\frac{\Delta H}{R T}+\frac{\Delta S}{R}
\end{aligned}
$$

where $K$ is the thermodynamic equilibrium constant, which is determined by plotting $\ln$ $\left(Q_{\mathrm{e}} / C_{\mathrm{e}}\right)$ versus $Q_{\mathrm{e}}$ and extrapolating to zero $Q_{\mathrm{e}}, 1 / n$ is the adsorption intensity of the resin, $R$ is the universal gas constant $(8.314 \mathrm{~J} /(\mathrm{mol} \cdot \mathrm{K}))$, and $T(\mathrm{~K})$ denotes the absolute temperature.

\section{Dynamic Adsorption and Desorption}

Dynamic adsorption and desorption experiments were performed via glass column chromatography. The glass columns $(2.5 \mathrm{~cm}$ by $40 \mathrm{~cm})$ were wet-packed with the previously selected resins (bed volumes of $150 \mathrm{~mL}$ ). Then, $10 \mathrm{~g}$ of crude extract (from Eucommia ulmoides leaves) was dissolved in $100 \mathrm{~mL}$ of distilled water (yielding a final concentration of $10 \mathrm{mg} / \mathrm{mL}$ ) and the solution was carefully loaded onto the top of the column. The column was left at a constant temperature overnight until the adsorption equilibrium was reached. An ethanol-water solution was used as the elution solvent, and the volume of each solution was 3.0 BV. During the dynamic desorption testing, an aliquot of distilled water, $20 \%$ (v/v) ethanol, 40\% (v/v) ethanol, 60\% (v/v) ethanol, $80 \%$ (v/v) ethanol, and $100 \%(\mathrm{v} / \mathrm{v})$ ethanol was loaded sequentially into the column at room temperature and eluted with a constant flow rate of $1.5 \mathrm{BV} / \mathrm{h}$. The different concentration eluents were collected separately, and each fraction was separately condensed at reduced pressure and $50{ }^{\circ} \mathrm{C}$, using a rotary evaporator. After evaporation, all the obtained fractions were lyophilized in a freeze-dryer, weighed, and stored at $-20{ }^{\circ} \mathrm{C}$ prior to further analysis.

\section{Acetylcholinesterase Inhibitory Activity}

The acetycholine esterase inhibitory activity was measured using Ellman's method (Sallam et al. 2016; Raza et al. 2019). The reaction substrate acetylthiocholine iodide 
(ACTI) can be hydrolyzed to acetate and thiocholine by AChE. Under neutral or alkaline conditions, thiocholine can react with DTNB, and the reaction product is yellow, and the absorbance of the solution can be detected via spectroscopy at $412 \mathrm{~nm}$. Briefly, $2.65 \mathrm{~mL}$ of a Tris/HCl buffer solution ( $\mathrm{pH} 8.0,1.0 \mathrm{M})$ and $100 \mu \mathrm{L}$ of the different concentration test sample solutions were added to $50 \mu \mathrm{L}$ of $0.85 \mathrm{U} / \mathrm{mL}$ acetylcholinesterase for each sample. The solutions were incubated at $37^{\circ} \mathrm{C}$ for $10 \mathrm{~min}$, then $100 \mu \mathrm{L}$ of $15 \mathrm{mM}$ DTNB was added and uniformly mixed. Subsequently, the reaction was continued at $37{ }^{\circ} \mathrm{C}$ for $20 \mathrm{~min}$, and then a $4 \%$ SDS solution was immediately added to stop the reaction. The absorbance of the resulting solution was recorded at $412 \mathrm{~nm}$, and the inhibition rate was calculated according to the absorbance of the different samples. The $\mathrm{IC}_{50}$ value can be calculated based on the curve obtained from the inhibition concentration and inhibition rate. In addition, to avoid the effects of changes in absorbance due to the color of the compound or spontaneous hydrolysis of the substrate, the absorbance of the solution should be deducted before adding the enzyme. All experiments were carried out under their own control condition and repeated three times.

\section{Docking Studies}

The docking experiment was used to investigate the binding affinity of the target compounds to the binding residues of the active site of AChE. The crystal structure of AChE (PDB: 6cqy) was downloaded from the RCSB Protein Data Bank (PDB) (https://www.rcsb.org/) and was used for the docking studies. All cofactors, ligands, and water molecules were removed from the protein structure before docking. The threedimensional structures of the ligands were obtained from PubChem data (NCBI, USA) (https://www.ncbi.nlm.nih.gov/), and the ligands structures should be saved in the mol2 format before use. Docking studies were carried out via SYBYL X 2.0, according to its operating methodology. When the docking experiment was completed, the degree of binding between the small molecules and proteins was estimated, which was based on the docking scores of the different small molecules and proteins.

\section{Statistical Analysis}

The test data was collected in Microsoft Excel 2010. All values are the means \pm SDs of the three independent experiments, and the figures were built in Origin 2019b (Originlab Corporation, Northampton, MA).

\section{RESULTS AND DISCUSSION}

\section{Static Adsorption and Desorption Investigation}

Macroporous resin is often used for the enrichment of natural active ingredients, because the macroporous resins not only has good enrichment ability, but also it is lower in terms of cost and can be recycled. In addition, ethanol and water are usually used as elution solvent, which are non-toxic, easy to recycle and environmentally friendly (Yang et al. 2016). It is worth nothing that the adsorption behavior of macroporous resins are not only associated with the polarity of the resin, but are also affected by the specific surface and the pore size, as well as being possibly affected by its physical properties, i.e., viscosity, density, etc. (Sandhu and Gu 2013). When the polarity of the adsorbent is similar to that of the adsorbed substance, it exhibits a high adsorption capacity. In addition, resins with a larger specific surface area and pore size usually have higher adsorption 
performance (Jia et al. 2011). In this study, the adsorption and desorption abilities of HPD100, HPD-300, HPD-600, D-3250, X-5, D-140, NKA-9, NKA-II, D-101, AB-8, S-8, and polyamide resins, in terms of the total extracted flavonoid content from EUO, were investigated, as shown in Fig. 1. The polyamide resin had the highest adsorption capacity $(19.19 \mathrm{mg} / \mathrm{g})$, followed by the AB-8 resin $(17.50 \mathrm{mg} / \mathrm{g})$, and the NKA-9 resin $(17.05 \mathrm{mg} / \mathrm{g})$. The results of the static adsorption experiments showed that the order of the resins, in terms of highest static adsorption, were as follows: S-8 was greater than HPD-300, which was greater than D-3250, which was greater than HPD-100, which was greater than HPD-600, which was greater than D-101, which was greater than X-5, which was greater than D-140, which was greater than NKA-II. Although the specific surface area of NKA-II was relatively large, the adsorption amount was lower in this resin type, which may suggest a relationship with its pore volume. Resins with a larger specific surface area exhibited an adsorption capacity of greater than $16 \mathrm{mg} / \mathrm{g}$. This also showed that due to the different structures of the target compounds, the adsorption capacity of the resin was closely related to the polarity, surface area, pore size, and volume of the adsorbent. This is probably due to flavonoid compounds containing a benzene ring structure. Therefore, the composition, physical structure, and polarity of the adsorbent were key factors that affected the adsorption performance.

The adsorption rate and adsorption capacity showed similar trends, and except for the NKA-II resin, the adsorption rate was approximately $80 \%$ or higher. The NKA-9 and AB-8 resins are cross-linked polystyrene resins, and they also showed a high adsorption capacity. In addition to the physical properties of the adsorbent, the effect of the polarity of the substance on the enrichment effect was also obvious. Among the selected resins, HPD-600, NKA-9, NKA-II, S-8, and polyamide were considered polar resins. Except for NKA-II, they all showed good adsorption capabilities, and in addition, the adsorption capacity of the weakly polar AB-8 resin was only lower than polyamide resins. These results indicated that the EUOL samples prepared by the method mentioned above were primarily polar or weakly polar flavonoid compounds.

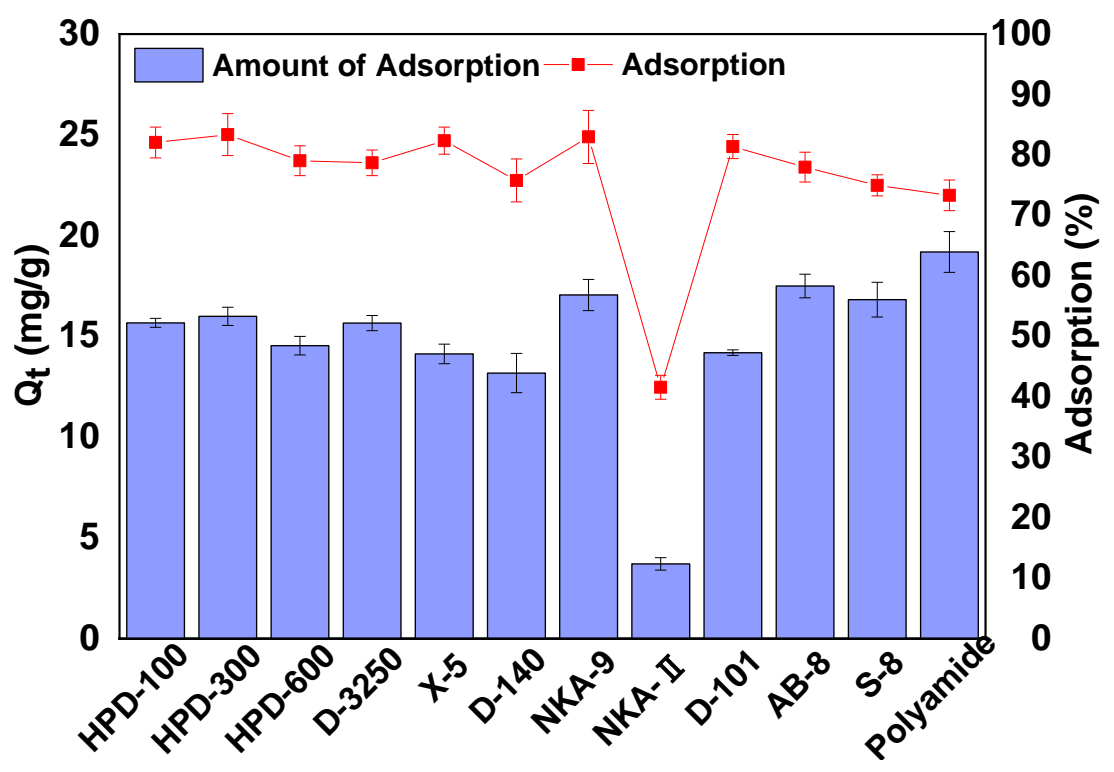

Fig. 1. Adsorption capacities and adsorption ratios of different resins in terms of flavonoids found in EUOL 


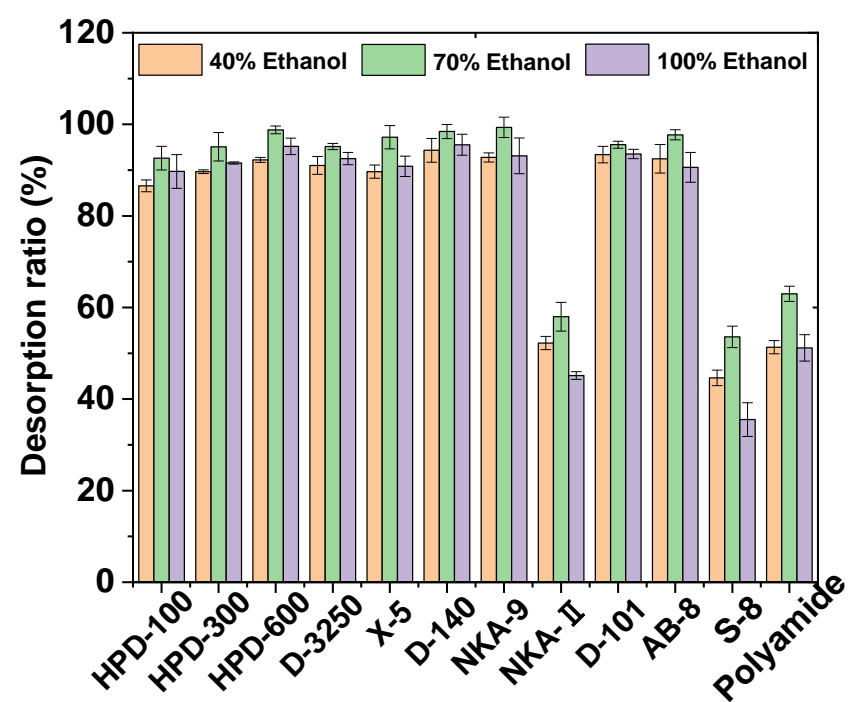

Fig. 2. Desorption ratios with different solvents and different resins in terms of flavonoids found in EUOL

The results of the static desorption experiment with different solvents are shown in Fig. 2. Except for NKA-II, S-8, and polyamide, the desorption rate of the other resins could reach greater than $80 \%$ for different desorption solvents. The desorption rate of the $70 \%$ ethanol solution was significantly higher than the desorption rate of the $40 \%$ and $100 \%$ ethanol solutions. Polyamide is considered to be a good adsorbent for enriching flavonoids, due to its special structure, but this is often related to the composition and structure of raw materials. In this experiment, polyamide resin showed a high adsorption capacity, but the desorption effect was not optimal compared to the other resins. The adsorption rate of S-8 was greater than $80 \%$, but the desorption of different solvents was relatively low. Among these resins, it may be that the resin is suitable for adsorbing flavonoids, but it is not easily desorbed due to its strong binding force. For the NKA-II resin, the adsorption rate and desorption rate were inferior to other resins in this experiment, especially the adsorption amount, which may not be suitable for enriching the flavonoids in the above-prepared samples, due to the special characteristics of the resin. Therefore, evaluation of the enrichment performance of a resin requires a comprehensive analysis of both the adsorption and desorption rates. The adsorption performance of macroporous resins were affected by many factors. When the polarities of the resin and the adsorbed compounds were the same or close to each other, a high adsorption efficiency could be achieved. In addition, resins with a large specific surface area generally achieved higher adsorption capabilities, but this also depended on the material composition and characteristics of the sample of interest (Deosarkar and Pangarkar 2004; Dong et al. 2015). Based on the above test results, resin AB-8 was selected for further analysis.

\section{Adsorption Kinetics}

The adsorption kinetics and equilibrium of the target component for the selected resins were investigated. The adsorption kinetic curves of the selected resins are shown in Fig. 3A. All of the resins exhibited a similar adsorption change with time, and the adsorption processes of all the resins were divided into three stages. During the first stage, the adsorption capacities of the selected resins showed a rapid increased trend within 20 
min, and although this process was fast adsorption, there were still differences between the different resins. During the second stage, the increase in the adsorption capacity of all the resins was slower than the first stage, and adsorption equilibrium was reached after $45 \mathrm{~min}$. The difference in adsorption capacities at this stage was more obvious for each resin. After 45 min of adsorption, the adsorption kinetics curve had basically stabilized. At this stage, the adsorption capacities of the different resins in terms of flavonoids could be clearly observed. Due to the differences in resin structure and composition, the adsorption effect on target substances was also different. The adsorption amount was between 8 to $12 \mathrm{mg} / \mathrm{g}$, which was also consistent with the previous test results. Rapid achievement of adsorption equilibrium can be regarded as beneficial for practical separation operations.

It was worth noting that the desorption rate was also an important factor in terms of the enrichment process. The kinetic desorption curves of the eight tested resins are shown in Fig. 3B. The desorption process could be basically divided into two stages. The concentration of the desorbed solution rapidly increased at the beginning, and then the change became balanced. Except for the D-3250 resin, the resins could complete rapid desorption within $40 \mathrm{~min}$, and HPD-300, HPD-600, AB-8, and X-5 could complete rapid desorption within $20 \mathrm{~min}$, with the AB-8 resin yielding the best results. The time for the desorption effect to reach equilibrium for the other resins were ranked as follows: NKA-9 was greater than D-101, which was greater than D-140. This result indicated that the adsorption and desorption of flavonoids by these resins were able to rapidly reach equilibrium.
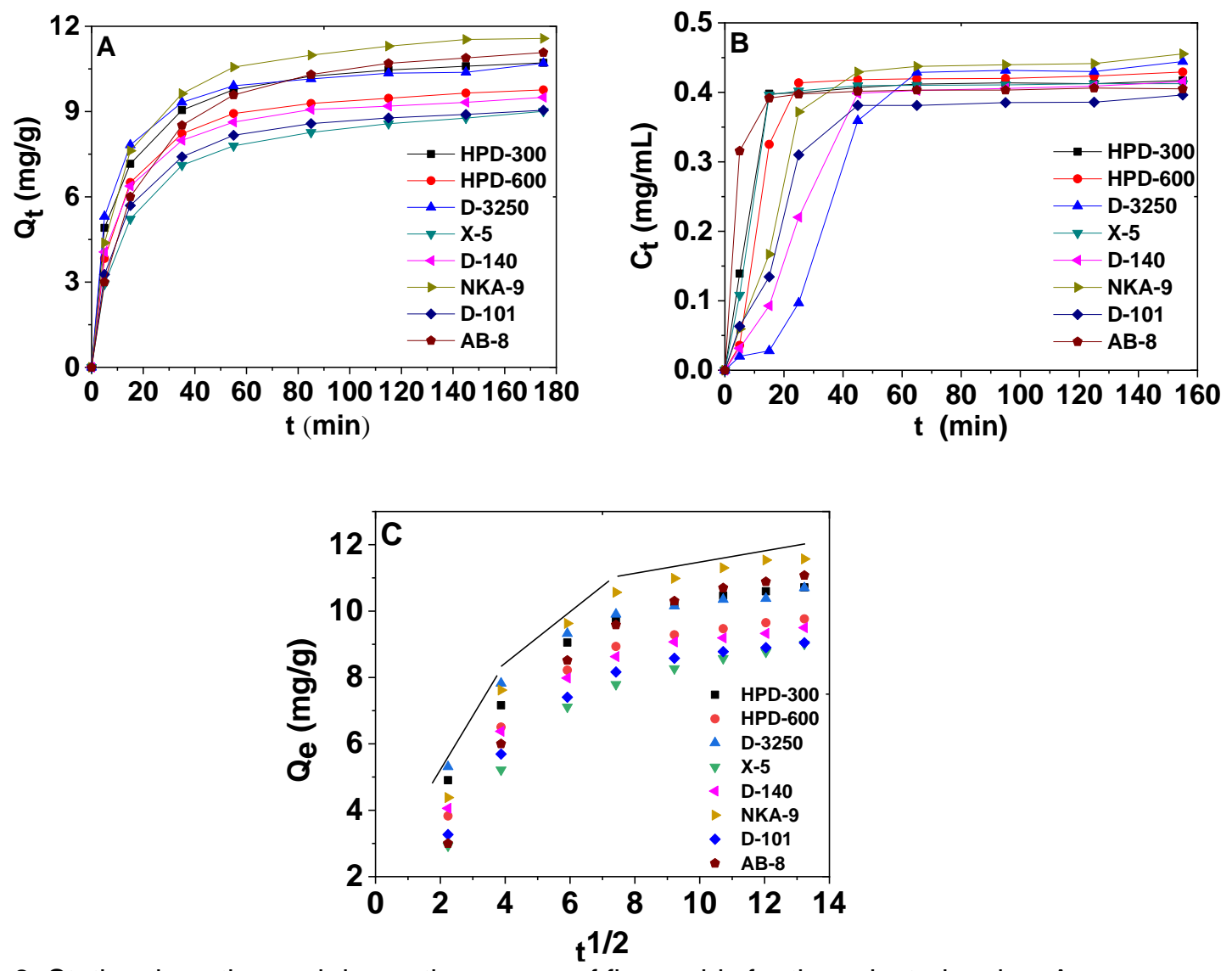

Fig. 3. Static adsorption and desorption curves of flavonoids for the selected resins: A adsorption curves; B - desorption curves; and C - intra-particle diffusion curves 
The adsorption kinetic curve is an important parameter in defining the adsorption efficiency and the equilibrium time, which is related to the adsorption mechanism in the process. Therefore, it is important to utilize adsorption kinetic models that fit the data obtained in the laboratory, so the contact time of the sorption reaction could be precisely governed. In this work, the pseudo first-order, pseudo second-order, and intra-particle diffusion kinetics models were applied to clarify the adsorption process. The fitting equations and kinetic parameters at $25^{\circ} \mathrm{C}$ and correlation coefficients of the pseudo-firstorder and pseudo-second-order kinetic models and particle diffusion were calculated and listed in Table 2. In the kinetic experiment, the pseudo-first-order model was primarily used to describe the initial stage of adsorption, while the second-order equation was used to predict the adsorption behavior of the entire adsorption process ( Lv et al. 2018). It was found that the pseudo-second-order kinetic model was better at depicting the kinetics of the adsorption of flavonoids onto resins, The coefficient of determination $\left(\mathrm{R}^{2}\right)$ of the pseudo second-order model was greater than that of the pseudo first-order model, which meant the pseudo second-order model had a better linear correlation with the adsorption kinetics, and the theoretical $Q_{\mathrm{e}}$ values estimated from the pseudo-second-order kinetic model were close to the measured results. This indicated that the pseudo-second-order model was better at explaining the flavonoid adsorption of the selected resins.

Table 2. Equations of the Pseudo-First-Order, Pseudo-Second-Order, and Particle Diffusion Kinetics Models and the Dynamic Parameters for Different Resins

\begin{tabular}{|c|c|c|c|c|}
\hline \multirow{2}{*}{ Resins } & \multirow{2}{*}{ Dynamic Equation } & \multicolumn{2}{|c|}{ Dynamic Parameters } & \multirow{2}{*}{$\begin{array}{l}\text { Coefficient of } \\
\text { Determination }\end{array}$} \\
\hline & & $Q_{e}(\mathrm{mg} / \mathrm{g})$ & $k$ & \\
\hline \multirow{3}{*}{$\begin{array}{l}\text { HPD- } \\
300\end{array}$} & Pseudo-first-order & 8.8995 & 0.0037 & 0.6929 \\
\hline & Pseudo-second-order & 10.8260 & 0.0149 & 0.9876 \\
\hline & Particle diffusion & 5.2429 & 0.4789 & 0.7980 \\
\hline \multirow{3}{*}{$\begin{array}{l}\text { HPD- } \\
600\end{array}$} & Pseudo-first-order & 8.2665 & 0.0039 & 0.6663 \\
\hline & Pseudo-second-order & 10.1958 & 0.0118 & 0.9998 \\
\hline & Particle diffusion & 4.4051 & 0.4704 & 0.7620 \\
\hline \multirow{3}{*}{ D-3250 } & Pseudo-first-order & 8.1042 & 0.0034 & 0.6412 \\
\hline & Pseudo-second-order & 10.7817 & 0.0178 & 0.9977 \\
\hline & Particle diffusion & 5.9113 & 0.4122 & 0.7440 \\
\hline \multirow{3}{*}{$X-5$} & Pseudo-first-order & 9.0789 & 0.0039 & 0.7412 \\
\hline & Pseudo-second-order & 9.3572 & 0.0097 & 0.9987 \\
\hline & Particle diffusion & 3.1860 & 0.4977 & 0.8254 \\
\hline \multirow{3}{*}{$\mathrm{D}-140$} & Pseudo-first-order & 6.9682 & 0.0044 & 0.6970 \\
\hline & Pseudo-second-order & 9.7040 & 0.0146 & 0.9971 \\
\hline & Particle diffusion & 4.4963 & 0.4357 & 0.7800 \\
\hline \multirow{3}{*}{ NKA-9 } & Pseudo-first-order & 9.7385 & 0.0041 & 0.6828 \\
\hline & Pseudo-second-order & 12.1729 & 0.0092 & 0.9999 \\
\hline & Particle diffusion & 5.0340 & 0.5772 & 0.7725 \\
\hline \multirow{3}{*}{ D-101 } & Pseudo-first-order & 8.6634 & 0.0037 & 0.6815 \\
\hline & Pseudo-second-order & 9.4841 & 0.0110 & 0.9995 \\
\hline & Particle diffusion & 3.7029 & 0.4672 & 0.7846 \\
\hline \multirow{3}{*}{ AB-8 } & Pseudo-first-order & 11.6440 & 0.0042 & 0.7351 \\
\hline & Pseudo-second-order & 12.1271 & 0.0054 & 0.9999 \\
\hline & Particle diffusion & 3.3067 & 0.6746 & 0.8243 \\
\hline
\end{tabular}

Adsorption kinetics may be controlled by the rate limiting step of the process and the adsorption mechanism. Generally, the experimental data fit via the intra-particle 
diffusion model was shown in Fig. 3C. With an increasing adsorption time, the diffusion curves of $\mathrm{Q}_{\mathrm{t}}$ versus $t^{1 / 2}$ presented multi-linearity and the adsorption process could be divided into three stages. The first stage $(0 \mathrm{~min}$ to $20 \mathrm{~min})$ exhibited boundary layer diffusion, where phenolic compound molecules diffused from the solvent to the surface of the resin particles. The second stage (20 $\mathrm{min}$ to $50 \mathrm{~min}$ ) was the gradual adsorption stage, when the internal diffusion had a rate limitation, and the third stage (50 min to $175 \mathrm{~min}$ ) was the final equilibrium stage. In this research, the plots of $\mathrm{Q}_{\mathrm{t}}$ versus $t^{1 / 2}$ were multi-linear, which was due to the broad pore size distribution of the selected resins. The first linear plot was ascribed to the adsorption of flavonoids over the shell and macropores, and therefore was the fastest adsorption stage. The second plot was due to the intra-particle diffusion through the mesopore. The third plot was attributed to diffusion through the micropores, which was followed with establishing the adsorption equilibrium (Liu et al. 2010; Duran et al. 2011).

Therefore, the adsorption performance of the macroporous resins in terms of flavonoids was associated with the synergistic effects of their chemical compositions and physical properties (Lv et al. 2018). The selected resins with larger specific surface areas possessed a slightly stronger desorption capacity than the other three resins, which suggested that the specific surface area might be one of the critical factors that affect the desorption process (Scordino et al. 2003). In summary, all the above results showed that the eight resins exhibited similar adsorption behaviors and possessed good adsorption capacities. This suggested that all the chemical constitutions of the eight resins were suitable for adsorption of the flavonoids found in Eucommia ulmoides Oliv. leaves. Meanwhile, the similar adsorption capacities of the eight resins indicated that both the chemical constituents and the physical structures were important in terms of the adsorption capabilities of the resins.

\section{Adsorption Isotherm}

Isothermal adsorption data can be used to investigate the affinity between the adsorbent and the adsorbate. The Langmuir and Freundlich equations are the most popular equations for evaluating adsorption isotherms due to their relative simplicity and reasonableness, and their accurate description (Buran et al. 2014). The Langmuir equation can be used to describe a monolayer adsorption, whereas the Freundlich equation, known as an empirical equation, can be used to describe a multilayer and heterogeneous surface adsorption (Yang et al. 2016). In addition, as a typical extended form of the Langmuir model, the Sips model investigates the high-energy heterogeneous adsorption characteristics of the adsorbent surface by introducing heterogeneous parameters. Therefore, based on the results obtained from the above kinetics, through comprehensive analysis of the adsorption and desorption performances, the NKA-9, AB-8, and HPD-300 resins were selected for further analysis. Meanwhile, the adsorption isotherm models, including the Langmuir, Freundlich, and Sips models, were selected to analyze the adsorption mechanisms of the selected resins in terms of flavonoids.

It can be seen from the experimental results that the adsorption capacity of these resins in terms of flavonoids found in EUOL was greater at $25{ }^{\circ} \mathrm{C}$ than at other temperatures. The different isotherm models for the NKA-9, AB-8, and HPD-300 resins were evaluated in this experiment, and the isotherm parameters for the adsorption capacity of flavonoids are shown in Table 3. 
Table 3. Parameters of the Langmuir, Freundlich, and Sips models For the Three Selected Resins

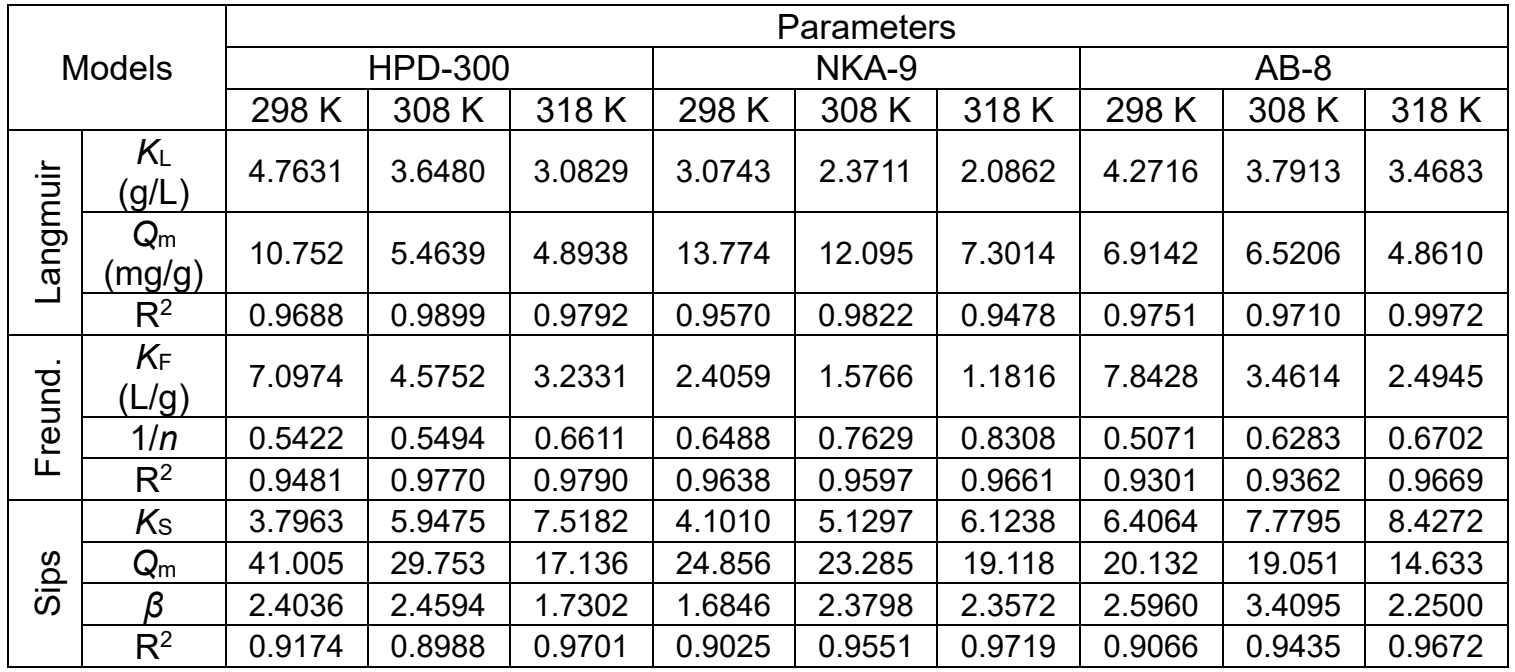

For the Langmuir equation, $K_{\mathrm{L}}$ is the key factor for the affinity of the binding site. During this adsorption process, as the temperature increased, the value of $K_{\mathrm{L}}$ decreased. However, the Freundlich model is often used to analyze the internal surface adsorption mechanism of solid-liquid to obtain the desired analysis results, however, its scope of application is not as extensive as the Langmuir model. The constants $K_{\mathrm{F}}$ and $n$ represent the indicators of adsorption capacity and intensity of the resins selected, respectively. A value of $1 / n$ between 0 and 1 indicated that this condition was favorable for the occurrence of adsorption, but when its value is greater than 1.0, the adsorption process is more difficult (Wu et al. 2016). As shown in Table 3, when the adsorption temperature was greater at 25 ${ }^{\circ} \mathrm{C}$, the value of $1 / n$ increased as the temperature increased. This indicated that high temperatures were not conducive to the adsorption of flavones by the selected resins. It also confirmed that temperature is a key factor affecting the amount of adsorption. Higher temperatures may accelerate the frequency of thermal motion of the molecules in the solution, thereby reducing the interaction between the target compound and the active sites on the resin, leading to a reduction in the amount of adsorption ( $\mathrm{Lv}$ et al. 2018). From the analysis results, it can be found that the Langmuir model had a good coefficient of determination at different temperatures compared with other models, which means that it can be used as a good adsorption model to reflect the adsorption equilibrium of the selected resins in terms of flavonoids. It also showed that the adsorption process involved singlelayer adsorption equivalent non-interacting monomeric sites, and it was also accompanied by other adsorption models. For different adsorption resins, the correlation coefficients of the different adsorption isothermal models were significantly different, and it was inferred that there are differences in the adsorption mechanisms of different resins in terms of EUOL, which is also an important reason for the differences in adsorption and desorption characteristics.

\section{Adsorption Thermodynamics}

Determining the adsorption thermodynamics is necessary to reveal in-depth information about the consumed energy and adsorbing mechanism. The adsorption behavior and mechanism for the adsorption process of flavonoids by the selected resins can 
be understood by thermodynamic parameters (Chen et al. 2014). The Gibbs free energy $(\Delta G)$, enthalpy changes $(\Delta H)$, and entropy changes $(\Delta S)$ were calculated and employed to describe the adsorption thermodynamics at different temperatures in this work (as shown in Table 4). For the selected resins, the adsorption free energy changes were negative, which implied the adsorption could occur spontaneously (Yousef et al. 2011). The negative $\Delta H$ values $(-3.75 \mathrm{~kJ} / \mathrm{mol},-3.44 \mathrm{~kJ} / \mathrm{mol}$, and $-3.25 \mathrm{~kJ} / \mathrm{mol})$ at different temperatures demonstrated that the adsorption process was of an exothermic nature (Gökmen and Serpen 2002). Since the $\Delta S$ values were negative for the three selected resins, this suggested that the randomness of the adsorption process decreased at the solid-liquid interface (Lv et al. 2016). The absolute value of the $\Delta H$, which was lower than $43 \mathrm{~kJ} / \mathrm{mol}$, indicated that the adsorption reaction was primarily controlled by physical adsorption rather than a chemical mechanism (Yang et al. 2016). This result declared that the predominant force between the adsorbate and adsorbent was primarily electrostatic (Wang et al. 2012).

Table 4. Thermodynamic Parameters of the Three Selected Resins on the Adsorption Process

\begin{tabular}{|c|c|c|c|c|}
\hline Resins & $T(\mathrm{~K})$ & $\Delta S(\mathrm{~J} /(\mathrm{mol} \mathrm{K}))$ & $\Delta H(\mathrm{~kJ} / \mathrm{mol})$ & $\Delta G(\mathrm{~kJ} / \mathrm{mol})$ \\
\hline \multirow{3}{*}{ HPD-300 } & 298 & \multirow{3}{*}{-1.87} & \multirow{3}{*}{-3.75} & -3.19 \\
\hline & 308 & & & -3.17 \\
\hline & 318 & & & -3.15 \\
\hline \multirow{3}{*}{ NKA-9 } & 298 & \multirow{3}{*}{-0.760} & \multirow{3}{*}{-3.44} & -3.22 \\
\hline & 308 & & & -3.21 \\
\hline & 318 & & & -3.20 \\
\hline \multirow{3}{*}{ AB-8 } & 298 & \multirow{3}{*}{-0.300} & \multirow{3}{*}{-3.25} & -3.16 \\
\hline & 308 & & & -3.16 \\
\hline & 318 & & & -3.15 \\
\hline
\end{tabular}

The negative $\Delta H$ values indicated that the adsorption process was exothermic, and that lower temperatures facilitated the adsorption process. As described above, the kinetic data of the flavonoids adsorbed by the selected resins had a good fit with the pseudo second-order model, which suggested the adsorption was chemisorption. However, the $\Delta H$ values $(-3.75,-3.44$, and $-3.25 \mathrm{~kJ} / \mathrm{mol})$ suggested a physisorption mechanism. This further indicated that flavonoids had multiple adsorption methods by the selected resins. The results of kinetic and thermodynamic analyses are consistent with those described in the recent articles, and most of the adsorption systems conforming to the pseudo-second-order kinetic model are actually governed by diffusion-controlled kinetics and reversible physical adsorption (Hubbe et al. 2019).

\section{Dynamic Adsorption and Desorption of the Columns for the Selected Resins}

Based on the above experimental results, the NKA-9 resin was selected for dynamic elution to obtain different elution fractions from the crude extract. The dynamic elutes for the EUOL flavonoids from the NKA-9 resin columns were constructed to further evaluate the composition and biological activity of different eluted samples. The detection results of the flavonoid content in different eluted fractions are presented in Table 5. The results of the gradient elution showed that the desorption amount in the different elution solutions changed as the ratio of ethanol to water in the eluent changed. The total flavonoid content was highest in the $60 \%$ eluted fraction $(483.9797 \pm 1.9961 \mathrm{mg} / \mathrm{g})$ compared to the other fractions and was ten times higher than the original crude extract. In addition, the $40 \%$ and 
$80 \%$ ethanol eluted fractions were also rich in flavonoids, but the flavonoid content in the $40 \%$ ethanol eluted fraction was higher than the $80 \%$ eluted fraction. Although the total flavonoid content in the $20 \%$ ethanol eluent was higher than the $100 \%$ elution sample, their content was relatively low compared to the other fractions. The flavonoid content was the lowest in the water eluting fraction, only $2.4721 \pm 0.5429 \mathrm{mg} / \mathrm{g}$. From the results, it was determined that $95 \%$ of the total flavonoid content was contained in the $40 \%, 60 \%$, and $80 \%$ ethanol elution fractions for all the eluted samples in this experiment. Thus, the $60 \%$ ethanol elution was selected to elute and collect the target compounds.

Table 5. Total Flavonoid Content and Enzyme Inhibitory Activity of the Fraction Eluted from the Column Packed with NKA-9 Resin

\begin{tabular}{|c|c|c|}
\hline Ethanol Concentration & $\begin{array}{c}\text { Content of Flavonoids } \\
(\mathrm{mg} / \mathrm{g})\end{array}$ & $\begin{array}{c}\text { Acetylcholinesterase Inhibition } \\
\text { IC50 }(\mathrm{mg} / \mathrm{mL})\end{array}$ \\
\hline Water & $2.4721 \pm 0.5429$ & - \\
\hline $20 \%$ ethanol & $23.8709 \pm 0.0396$ & $3.3359 \pm 0.0634$ \\
\hline $40 \%$ ethanol & $330.3241 \pm 1.6820$ & $0.7712 \pm 0.0219$ \\
\hline $60 \%$ ethanol & $483.9797 \pm 1.9961$ & $0.5893 \pm 0.0079$ \\
\hline $80 \%$ ethanol & $304.0226 \pm 2.0938$ & $0.9966 \pm 0.0489$ \\
\hline $100 \%$ ethanol & $16.0071 \pm 0.5482$ & $4.6278 \pm 0.1090$ \\
\hline Crude extract & $48.2780 \pm 1.0069$ & $1.4480 \pm 0.0022$ \\
\hline
\end{tabular}

Table 5 displays the acetylcholinesterase inhibitory effect of the different elution components. As shown by the results, the trends of the acetylcholinesterase inhibition activity for the different eluted fractions was similar to the flavonoid content trends. The greatest inhibitory effects on the enzyme activity were by the $40 \%, 60 \%$, and $80 \%$ ethanoleluting fractions, and the $60 \%$ eluting component had the greatest inhibitory effect on the enzymes. This also demonstrated that as a potential inhibitor of enzyme activity, it could be effectively enriched under these conditions. Enrichment to obtain a higher target ingredient content or to obtain a synergistic effect between different ingredients can effectively inhibit the activity of acetylcholinesterase.

Based on the above experimental results, the NKA-9 resin had good enrichment effects on the flavonoid found in EUOL. Considering the content and biological activity of the target flavonoids in the different eluted fractions, a stepwise elution scheme should be used when preparing the required target components, such as the following test steps: the substances that were not adsorbed by the resin are removed using deionized water, then additional undesired components were removed via elution with $20 \%$ ethanol. Elution fractions can then be collected in stages and further analyzed.

In order to further understand the effects of the flavonoids found in EUOL and acetylcholinesterase, the primary flavonoid types were determined. Based on previous literature reports (He et al. 2014; Hirata et al. 2014; Zhu and Sun 2018), EUOL flavonoids primarily included rutin, quercetin, kaempferol, hypericin, terpinein, astragalin, isoquercetin, isorhamnetin, isorhamnetin-3-o- $\beta$-D-glucoside, kaempferol-3-O-rutinoside, etc., and the primarily flavonoid aglycones were quercetin and kaempferol. Therefore, in this experiment, molecular docking technology was used to investigate the affinity of the main flavonoids in EUOL with acetylcholinesterase.

Because the sample components found in the different eluting fractions were complicated, different compounds could synergistically inhibit acetylcholinesterase activity. Based on the known flavonoids found in Eucommia ulmoides, the scoring results 
of the enzyme protein molecule dockings are shown in the Table 6 , and the results suggested that the binding degree of different flavonoid compounds and enzymes were drastically different. Kaempferol-3-O-rutinoside had the highest score, followed by Isoquercetin, while hypericin had the lowest docking score (the docking results are shown in Fig. 4.). The scores for the other compounds ranged from 3 to 5 . For the flavone aglycon, the docking score of quercetin was higher than kaempferol. For compounds with flavone aglycon linked to different glycoside groups, the degree of docking affinity was different. This also showed that due to the different structures of the compounds, when docking with macromolecules, the conformation and interaction force at the active site would be different, so the degree of binding to the protein molecule would eventually be different.

Table 6. Structural Information and Molecular Docking Scores of the Primary Flavonoids in EUOL

\begin{tabular}{|c|c|c|c|c|}
\hline No. & Molecular Formula & Molecular Weight & Structure & Scores \\
\hline 1 & Rutin & 610.5 & $\mathrm{C}_{27} \mathrm{H}_{30} \mathrm{O}_{16}$ & 4.9264 \\
\hline 2 & Quercetin & 302.2 & $\mathrm{C}_{15} \mathrm{H}_{10} \mathrm{O}_{7}$ & 4.6462 \\
\hline 3 & Kaempferol & 286.2 & $\mathrm{C}_{15} \mathrm{H}_{10} \mathrm{O}_{6}$ & 3.0288 \\
\hline 4 & Hypericin & 504.4 & $\mathrm{C}_{30} \mathrm{H}_{16} \mathrm{O}_{8}$ & 1.3382 \\
\hline 5 & $\begin{array}{c}\text { Quercetin-3-O-D- } \\
\text { glucopyranoside }\end{array}$ & 464.4 & $\mathrm{C}_{21} \mathrm{H}_{20} \mathrm{O}_{12}$ & 3.7143 \\
\hline 6 & Astragalin & 448.4 & $\mathrm{C}_{21} \mathrm{H}_{20} \mathrm{O}_{11}$ & 3.9688 \\
\hline 7 & Isoquercetin & 464.4 & $\mathrm{C}_{21} \mathrm{H}_{20} \mathrm{O}_{12}$ & 5.3324 \\
\hline 8 & Isorhamnetin & 316.3 & $\mathrm{C}_{16} \mathrm{H}_{12} \mathrm{O}_{7}$ & 4.4370 \\
\hline 9 & $\begin{array}{c}\text { Isorhamnetin-3-O- } 3-D- \\
\text { glucoside }\end{array}$ & 478.4 & $\mathrm{C}_{22} \mathrm{H}_{22} \mathrm{O}_{12}$ & 3.1659 \\
\hline 10 & Kaempferol-3-O-rutinoside & 594.5 & $\mathrm{C}_{27} \mathrm{H}_{30} \mathrm{O}_{15}$ & 6.4614 \\
\hline
\end{tabular}
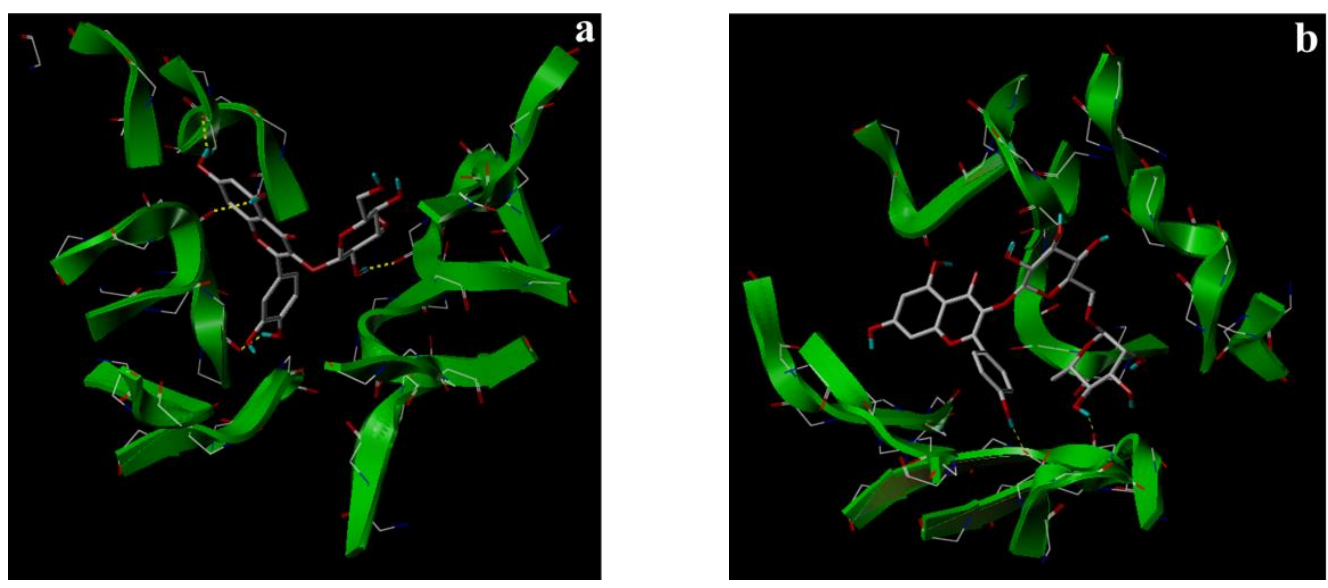

Fig. 4. Molecular docking of (a) Isoquercetin and (b) Kaempferol-3-O-rutinoside with acetylcholinesterase

\section{CONCLUSIONS}

1. The adsorption and desorption characteristics of twelve macroporous resins in terms of the flavonoids found in Eucommia ulmoides Oliv. were investigated. Eight resins were selected for further analysis through static adsorption and desorption experiments.

2. The pseudo-second-order kinetics and Langmuir isotherm models were demonstrated 
to be appropriate to describe the entire exothermic and physical adsorption processes of the antioxidants by the resins. Furthermore, according to the research results of adsorption and desorption characteristics, NKA-9 resin and 60\% ethanol were confirmed to be the best sorbent and desorption solvent, respectively. In addition, the Eucommia ulmoides Oliv. 60\% ethanol eluted fraction using NKA-9 resin possessed a better inhibitory effect of acetylcholinesterase when compared to the other fractions. Nearly $90 \%$ of the total flavonoids were detected in the $40 \%, 60 \%$, and $80 \%$ eluent fractions.

3. The difference in binding between the different flavonoid compounds and the target protein could be found through molecular docking technology, and this method could also facilitate screening for excellent enzyme activity inhibitors.

4. The enrichment process of the flavonoids found in Eucommia ulmoides Oliv. using NKA-9 resin was efficient and economical and has a great industrial production potential. Meanwhile, the production of highly concentrated flavonoids might expand the applications of E. ulmoides as a bioactive ingredient in functional foods and the pharmaceutical industry.

\section{ACKNOWLEDGEMENTS}

This study was financially supported by the GDAS' Project of Science and Technology Development (2019GDASYL-0103024, 2019GDASYL-0502003), the National Natural Science Foundation of China (31660181), and funded by the China Postdoctoral Science Foundation (2019M653099).

\section{REFERENCES CITED}

Bai, M.-M., Shi, W., Tian, J.-M., Lei, M., Kim, J. H., Sun, Y. N., Kim, Y. H., and Gao, J.-M. (2015). "Soluble epoxide hydrolase inhibitory and anti-inflammatory components from the leaves of Eucommia ulmoides Oliver (Duzhong)," Journal of Agricultural and Food Chemistry 63(8), 2198-2205. DOI: 10.1021/acs.jafc.5b00055

Buran, T. J., Sandhu, A. K., Li, Z., Rock, C. R., Yang, W. W., and Gu, L. W. (2014). "Adsorption/desorption characteristics and separation of anthocyanins and polyphenols from blueberries using macroporous adsorbent resins," Journal of Food Engineering 128, 167-173. DOI: 10.1016/j.jfoodeng.2013.12.029

Cho, S., Hong, R., Yim, P., Yim, P., Yeom, M., Lee, B., Yang, W. M., Hong, J., Lee, H. S., and Hahm, D.-H. (2018). "An herbal formula consisting of Schisandra chinensis (Turcz.) Baill, Lycium chinense Mill and Eucommia ulmoides Oliv alleviates disuse muscle atrophy in rats," Journal of Ethnopharmacology 213, 328-339. DOI: 10.1016/j.jep.2017.10.008

Chen, R., Yang, Q., Zhong, Y., Li, X., Liu, Y., Li, X.-M., Du, W.-X., and Zeng, G.-M. (2014). "Sorption of trace levels of bromate by macroporous strong base anion exchange resin: Influencing factors, equilibrium isotherms and thermodynamic studies," Desalination 344, 306-312. DOI: 10.1016/j.desal.2014.04.001

Deosarkar, S. P., and Pangarkar, V. G. (2004). "Adsorptive separation and recovery of organics from PHBA and SA plant effluents," Separation and Purification 
Technology 38(3), 241-254. DOI: 10.1016/j.seppur.2003.11.012

Dong, Y., Zhao, M., Sun-Waterhouse, D., Zhuang M., Chen, H., Feng, M., and Lin, L. (2015). "Absorption and desorption behaviour of the flavonoids from Glycyrrhiza glabra L. leaf on macroporous adsorption resins," Food Chemistry 168, 538-545. DOI: 10.1016/j.foodchem.2014.07.109

Duran, C., Ozdes, D., Gundogdu, A., and Senrurk, H. B. (2011). "Kinetics and isotherm analysis of basic dyes adsorption onto almond shell (Prunus dulcis) as a low cost adsorbent," Journal of Chemical and Engineering Data 56(5), 2136-2147. DOI: $10.1021 / \mathrm{je} 101204 \mathrm{j}$

Gökmen, V., and Serpen, A. (2002). "Equilibrium and kinetic studies on the adsorption of dark colored compounds from apple juice using adsorbent resin," Journal of Food Engineering 53(3), 221-227. DOI: 10.1016/S0260-8774(01)00160-1

He, X. R., Wang, J. H., Li, M. X., Hao, D. J., Yang, Y., Zhang, C. L., He, R., and Tao, R. (2014). "Eucommia ulmoides Oliv.: Ethnopharmacology, phytochemistry and pharmacology of an important traditional Chinese medicine," Journal of Ethnopharmacology 151(1), 78-92. DOI: 10.1016/j.jep.2013.11.023

Hirata, T., Ikeda, T., Fujikawa, T., and Sansei, N. (2014). "The chemistry and bioactivity of Eucommia ulmoides Oliver leaves," Studies in Natural Products Chemistry 41, 225-260. DOI: 10.1016/b978-0-444-63294-4.00008-5

Hosoo, S., Koyama, M., Watanabe, A., Ishida, R., Hirata, T., Yamaguchi, Y., Yamasaki, H., Wada, K., Higashi, Y., and Nakamura, K. (2017). "Preventive effect of Eucommia leaf extract on aortic media hypertrophy in Wistar-Kyoto rats fed a high-fat diet," Hypertension Research 40, 546-551. DOI: 10.1038/hr.2016.189

Hou, M., Hu, W., Xiu, Z., Shi, Y., Hao, K., Cao, D., Guan, Y., and Yin, H. (2020). "Efficient enrichment of total flavonoids from Pteris ensiformis Burm. extracts by macroporous adsorption resins and in vitro evaluation of antioxidant and antiproliferative activities," Journal of Chromatography B 1138, 121960. DOI: 10.1016/j.jchromb.2019.121960

Hubbe, M. A., Azizian, S., and Douven, S. (2019). "Implications of apparent pseudosecond-order adsorption kinetics onto cellulosic materials. A review," BioResources 14(3), 7582-7626. DOI: 10.15376/biores.14.3.7582-7626

Jia, G., and Lu, X. (2008). "Enrichment and purification of madecassoside and asiaticoside from Centella asiatica extracts with macroporous resins," Journal of Chromatography A 1193(1-2), 136-141. DOI: 10.1016/j.chroma.2008.04.024

Jia, D., Li, S., and Gu, Z. (2011). "Preparative isolation of flavonoids from mulberry (Morus alba L.) leaves by macroporous resin adsorption," Journal of Food Process Engineering 34(4), 1319-1337. DOI: 10.1111/j.1745-4530.2009.00427.x

Jiang, H., Li, J., Chen, L., and Wang, Z. (2020). “Adsorption and desorption of chlorogenic acid by macroporous adsorbent resins during extraction of Eucommia ulmoides leaves," Industrial Crops and Products 149, 112336. DOI: 10.1016/j.indcrop.2020.112336

Jin, X., Liu, M., Chen, Z., Mao, R., Xiao, Q., Gao, H., and Wei, M. (2015). “Separation and purification of epigallocatechin-3-gallate (EGCG) from green tea using combined macroporous resin and polyamide column chromatography," Journal of ChromatographyB 1002, 113-122. DOI: 10.1016/j.jchromb.2015.07.055

Kim, H. Y., Moon, B. H., Lee, H. J., and Choi, D. H. (2004). "Flavonol glycosides from the leaves of Eucommia ulmoides O. with glycation inhibitory activity," Journal of Ethnopharmacology 93(2-3), 227-230. DOI: 10.1016/j.jep.2004.03.047 
Kwon, S.-H., Lee, H.-K., Kim, J.-A., Hong, S.-I., Kim, S.-Y., Jo, T.-H., Park, Y.-I., Lee, C.-K., Kim, Y.-B., Lee, S.-Y., et al. (2011). "Neuroprotective effects of Eucommia ulmoides Oliv. bark on amyloid beta25-35-induced learning and memory impairments in mice," Neuroscience Letters 487(1), 123-127. DOI: 10.1016/j.neulet.2010.10.042

Lee, M.-K., Kim, M.-J., Cho, S.-Y., Park, S. A., Park, K.-K., Jung, U. J., Park, H.-M., and Choi, M.-S. (2005). "Hypoglycemic effect of Du-zhong (Eucommia ulmoides Oliv.) leaves in streptozotocin-induced diabetic rats," Diabetes Research and Clinical Practice 67(1), 22-28. DOI: 10.1016/j.diabres.2004.05.013

Li, Q., Feng, Y., He, W., Wang, L., Wang, R., Dong, L., Wang, C. (2017). "Postscreening characterisation and in vivo evaluation of an anti-inflammatory polysaccharide fraction from Eucommia ulmoides," Carbohydrate Polymers 169, 304-314. DOI: 10.1016/j.carbpol.2017.04.034

Li, X., Zhu, W., Yang, L., Fei, D., Fan, J., Du, L., and Liu, Y. (2013). "Evaluation of the sedative and hypnotic effects of eucommiol in Eucommia," Natural Product Research 27(18), 1657-1659. DOI: 10.1080/14786419.2012.746343

Liu, C., Jiao, R., Yao, L., Zhang, Y., Lu, Y., Tan, R. (2016). “Adsorption characteristics and preparative separation of chaetominine from Aspergillus fumigatus mycelia by macroporous resin," Journal of Chromatography B 1015-1016, 135-141. DOI: 10.1016/j.jchromb.2016.02.027

Liu, Q.-S., Zheng, T., Wang, P., Jiang J.-P., and Li, N. (2010). “Adsorption isotherm, kinetic and mechanism studies of some substituted phenols on activated carbonfibers," Chemical Engineering Journal 157(2-3), 348-356. DOI: 10.1016/j.cej.2009.11.013

Luo, D., Or, T. C. T., Yang, C. L. H., and Lau, A. S. Y. (2014). "Anti-inflammatory activity of iridoid and catechol derivatives from Eucommia ulmoides Oliver," ACS Chemical Neuroscience 5(9), 855-866. DOI: 10.1021/cn5001205

Lv, C., Yang, J., Liu, R., Lu, Q., Ding, Y., Zhang, J., and Deng, J. (2018). “A comparative study on the adsorption and desorption characteristics of flavonoids from honey by six resins," Food Chemistry 268, 424-430. DOI: 10.1016/j.foodchem.2018.06.100

Meda, A., Lamien, C. E., Romito, M., Millogo, J., and Nacoulma, O. G. (2005). "Determination of the total phenolic, flavonoid and proline contents in Burkina Fasan honey, as well as their radical scavenging activity," Food Chemistry 91(3), 571-577. DOI: 10.1016/j.foodchem.2004.10.006

Pompeu, D. R., Moura, F. G., Silva, E. M., and Rogez, H. (2010). "Equilibria, kinetics, and mechanisms for the adsorption of four classes of phenolic compounds onto synthetic resins," Separation Science and Technology 45(5), 700-709. DOI: 10.1080/01496390903562274

Raza, M. A., Fatima, K., Saqib, Z., Maurin, J. K., and Budzianowski, A. (2019). "Designing of diamino based esterases inhibitors; synthesis, characterization, density functional theory and molecular modeling," Journal of Molecular Structure 1195, 712-722. DOI: 10.1016/j.molstruc.2019.06.021

Sallam, A., Mira, A., Ashour, A., and Shimizu, K. (2016). "Acetylcholine esterase inhibitors and melanin synthesis inhibitors from Salvia officinalis," Phytomedicine 23(10), 1005-1011. DOI: 10.1016/j.phymed.2016.06.014

Sandhu, A. K., and Gu, L. (2013). "Adsorption/desorption characteristics and separation of anthocyanins from Muscadine (Vitis rotundifolia) juice pomace by use of macroporous adsorbent resins," Journal of Agricultural and Food Chemistry 61(7), 1441-1448. DOI: $10.1021 /$ jf3036148 
Scordino, M., Di Mauro, A., Passerini, A., and Maccarone, E. (2003). "Adsorption of flavonoids on resins: Hesperidin," Journal of Agricultural and Food Chemistry 51 (24), 6998-7004. DOI: 10.1021/jf034496q

State Pharmacopoeia Commission of the PRC (2015). Pharmacopoeia of the People's Republic of China (Vol. 1), China Medical Science and Technology Press, Beijing, China. p. 154.

Tanase, C., Cosarcă, S., and Muntean, D. L. (2019). "A critical review of phenolic compounds extracted from the bark of woody vascular plants and their potential biological activity," Molecules 24, 1182. DOI: 10.3390/molecules24061182

Tanase, C., Domokos, E., Cosarca, S., Miklos, A., Imre, S., Domokos, J., and Dehelean, C. A. (2018). "Study of the ultrasound-assisted extraction of polyphenols from beech (Fagus sylvatica L.) bark," BioResources 13(2), 2247-2267. DOI: 10.15376/biores.13.2.2247-2267

Wang, X., Peng, M. J., Wang, Z. H., Yang, Q. L., and Peng, S. (2020). “Ultrasoundmicrowave assisted extraction of flavonoid compounds from Eucommia ulmoides leaves and an evaluation of their antioxidant and antibacterial activities" Archives of Biological Sciences https://publons.com/publon/28391734/; DOI: 10.2298/ABS191216015W

Wang, X., Deng, R., Jin, X., and Huang, J. (2012). “Gallic acid modified hyper-crosslinked resin and its adsorption equilibria and kinetics toward salicylic acid from aqueous solution," Chemical Engineering Journal 191, 195-201. DOI: 10.1016/j.cej.2012.03.003

Wu, J., Pistolozzi, M., Liu, S., and Tan, W. (2020). "Design, synthesis and biological evaluation of novel carbamates as potential inhibitors of acetylcholinesterase and butyrylcholinesterase," Bioorganic \& Medicinal Chemistry 28(5), 115324. DOI: 10.1016/j.bmc.2020.115324

Wu, S., Wang, Y., Gong, G., Li, F., Ren, H., and Liu, Y. (2015). “Adsorption and desorption properties of macroporous resins for flavonoids from the extract of Chinese wolfberry (Lycium barbarum L.)," Food and Bioproducts Processing 93, 148-155. DOI: 10.1016/j.fbp.2013.12.006

Wu, Y., Zhang, L., Mao, J., Liu S., Huang, J., You, Y., and Mei, L. (2016). "Kinetic and thermodynamic studies of sulforaphane adsorption on macroporous resin," Journal of Chromatography B 1028, 231-236. DOI: 10.1016/j.jchromb.2016.06.035

Xing, Y.-F., He, D., Wang, Y., Zeng, W., Zhang, C., Lu, Y., Su, N., Kong, Y.-H., and Xing, X.-H. (2019). "Chemical constituents, biological functions and pharmacological effects for comprehensive utilization of Eucommia ulmoides Oliver," Food Science and Human Wellness 8(2), 177-188. DOI: 10.1016/j.fshw.2019.03.013

Xiong, N., Yu, R., Chen, T., Xue, Y.-P., Liu, Z.-Q., and Zheng, Y.-G. (2019). "Separation and purification of L-methionine from $E$. coli fermentation broth by macroporous resin chromatography," Journal of Chromatography B 1110-1111, 108115. DOI: 10.1016/j.jchromb.2019.02.016

Xu, Z., Tang, M., Li, Y., Liu, F., Li, X., Dai, R. (2010). “Antioxidant properties of Duzhong (Eucommia ulmoides Oliv.) extracts and their effects on color stability and lipid oxidation of raw pork patties," Journal of Agricultural and Food Chemistry 58(12), 7289-7296. DOI: 10.1021/jf100304t

Yang, Q., Zhao, M., and Lin, L. (2016). “Adsorption and desorption characteristics of adlay bran free phenolics on macroporous resins," Food Chemistry 194, 900-907. 
DOI: $10.1016 /$ j.foodchem.2015.08.070

Yousef, R. I., El-Eswed, B., and Al-Muhtaseb, A. H. (2011). "Adsorption characteristics of natural zeolites as solid adsorbents for phenol removal from aqueous solutions: Kinetics, mechanism, and thermodynamics studies," Chemical Engineering Journal 171(3), 1143-1149. DOI: 10.1016/j.cej.2011.05.012

Zhang, W., Fujikawa, T., Mizuno, K., Ishida, T., Ooi, K., Hirata, T., and Wada, A. (2012). "Eucommia leaf extract prevents OVX-induced osteoporosis and obesity in rats," The American Journal of Chinese Medicine 40(4), 735-752. DOI: 10.1142/S0192415X12500553

Zhou, Y., Liang, M., Li, W., Li, K., Li, P., Hu, Y., and Yang, Z. (2009). "Protective effects of Eucommia ulmoides Oliv. bark and leaf on amyloid $\beta$-induced cytotoxicity," Environmental Toxicology and Pharmacology 28(3), 342-349. DOI: 10.1016/j.etap.2009.05.012

Zhong, J.-L., Muhammad N, Gu, Y.-C., and Yan, W.-D. (2019). "A simple and efficient method for enrichment of cocoa polyphenols from cocoa bean husks with macroporous resins following a scale-up separation," Journal of Food Engineering 243, 82-88. DOI: $10.1016 /$ j.jfoodeng.2018.08.023

Zhu, M.-Q. and Sun, R.-C. (2018). "Eucommia ulmoides Oliver: A potential feedstock for bioactive products," Journal of Agricultural and Food Chemistry 66(22), 54335438. DOI: $10.1021 /$ acs.jafc.8b01312

Zhu, M.-Q., Wen, J.-L., Zhu, Y.-H., Su, Y.-Q., and Sun, R. C. (2016). "Isolation and analysis of four constituents from barks and leaves of Eucommia ulmoides Oliver by a multi-step process," Industrial Crops and Products 83, 124-132. DOI: 10.1016/j.indcrop.2015.12.049

Article submitted: Jun 23, 2020; Peer review completed: September 19, 2020; Revised version received and accepted: November 6, 2020; Published: November 24, 2020.

DOI: 10.15376/biores.16.1.470-491 